\title{
Estados tempranos de Echinoidea en canal Lagreze, Islas Guaitecas, sur de Chile
}

\author{
Early stages of Echinoidea in Lagreze Channel, Guaitecas Islands, southern Chile \\ Carlos Molinet ${ }^{1,3,4}$, Claudia Herrera ${ }^{1}$, Paulina Gebauer ${ }^{2}$, \\ Mauricio F. Landaeta ${ }^{3,5}$ y Carlos A. Moreno ${ }^{6,7}$ \\ ${ }^{1}$ Instituto de Acuicultura, Universidad Austral de Chile, sede Puerto Montt, Los Pinos s/n, \\ Balneario Pelluco, Casilla 1327, Puerto Montt, Chile \\ ${ }^{2}$ Centro I Mar, Universidad Los Lagos, Camino Chinquihue, $\mathrm{km}$ 6, Casilla 557, Puerto Montt, Chile \\ ${ }^{3}$ Centro de Investigación en Nutrición, Tecnología de Alimentos y Sustentabilidad, CIEN Austral, \\ Los Pinos s/n, Balneario Pelluco, Casilla 1327, Puerto Montt, Chile \\ ${ }^{4}$ Centro Trapananda, Universidad Austral de Chile, Portales 73, Coyhaique, Chile \\ ${ }^{5}$ Facultad de Ciencias del Mar y de Recursos Naturales, Universidad de Valparaíso, \\ Valparaíso, Casilla 5080, Reñaca, Viña del Mar, Chile \\ ${ }^{6}$ Instituto de Ecología y Evolución, Universidad Austral de Chile, Casilla 567, Valdivia, Chile \\ ${ }^{7}$ Centro de Investigaciones del Ecosistema Patagónico (CIEP), Bilbao 449, Coyhaique, Chile \\ cmolinet@uach.cl
}

\begin{abstract}
The limited publications on the dynamics of Echinoids on the coast of Chile, despite its ecological significance to marine benthic communities, have hindered the understanding of the importance of this group on benthic communities and the proposal of appropriate conservation and management measures. The objective of this work was to study the vertical and temporal variability of early stages of Echinoids during spring-summer period in a southern channel of southern Chile and associate it, in the particular case of Loxechinus albus, with the reproductive status of adults individuals. For this, i) daily samples of plankton in three depth strata were collected, every 15 days from October 2005 to February 2006, ii) postsettlers on artificial substrates were monitored and iii) the gonad somatic index was obtained for adult specimens of $L$. albus. Planktonic larval stages of Arbacia dufresnei, Loxechinus albus, Pseudechinus magellanicus and Ophiuroidea, were identified, as well as Ophiuroidea and L. albus post-settlement. Larvae of L. albus in plankton coincided with a decrease of gonad index in adults of this species, whereas post-settlers were observed 15 days after the longest period of larval density in the plankton. Although information on A. dufresnei and P. magellanicus reproduction in adults was not collected, autumn-winter period has been reported as the period of maximum larval abundance. Echinoids larvae were observed in restricted periods, more frequently on the sea surface at night and in low density compared with previous studies.
\end{abstract}

Key words: Larval density, vertical distribution, post-settlers, sea urchin, gonad index
Resumen.- Pese a la importancia ecológica de la clase Echinoidea para las comunidades bentónicas marinas, las publicaciones sobre la dinámica de estas especies en la costa de Chile son escasas, lo que dificulta la comprensión de la importancia de este grupo en las comunidades bentónicas y la proposición de medidas de conservación y/o manejo. El objetivo de este trabajo fue estudiar la variabilidad vertical y temporal de estadíos tempranos de Echinoidea durante primavera-verano en un canal del sur de Chile y asociarlo, en el caso particular de Loxechinus albus, con el estado reproductivo de ejemplares adultos. Para esto i) se recolectó muestras diarias de plancton en tres estratos de profundidad, cada 15 días entre octubre de 2005 y febrero de 2006, ii) se monitoreó post asentados en sustratos artificiales y iii) se obtuvo el índice gonadosomático para ejemplares adultos de L. albus. Se identificaron estadios larvales planctónicos de Arbacia dufresnei, Loxechinus albus, Pseudechinus magellanicus y de Ophiuroidea, y post asentados de Ophiuroidea y L. albus. Larvas de L. albus en el plancton coincidieron con una disminución del IGS en ejemplares adultos de esta especie, mientras que post asentados se observaron luego de 15 días del mayor periodo de densidad larval en el plancton. Aunque para A. dufresnei y P. magellanicus no se recolectó información sobre reproducción en adultos, se ha reportado otoño-invierno como el periodo de máxima abundancia larval. Larvas de Echinoidea fueron observadas en periodos restringidos, más frecuentes en superficie, durante la noche, y en baja densidad comparada con estudios anteriores.

Palabras clave: Densidad larval, distribución vertical, post asentados, erizos, índice gonadosomático 


\section{Introducción}

Entre los invertebrados marinos bentónicos, los organismos pertenecientes al phylum Echinodermata han sido descritos como uno de los grupos dominantes en las comunidades submareales bentónicas del océano Pacífico, debido a su alta contribución en la riqueza de especies. Este grupo ha sido ampliamente estudiado en aspectos como biología reproductiva, parámetros de historia de vida, ecología y acuicultura entre otros tópicos (Pennington 1985, Pennington \& Emlet 1986, Lawrence 2001, McBride et al. 2004, Nishizaki \& Ackerman 2004, Basch \& Tegner 2007).

Particularmente, en Chile se reconoce la influencia directa e indirecta de estas especies en la estructuración de las comunidades sublitorales (Arrau 1958, Ojeda \& Santelices 1984, Vásquez et al. 1984, Mutschke \& Ríos 2006). Otros aspectos estudiados sobre este grupo se han centrado principalmente en la historia de vida y ecología de Loxechinus albus y Pseudoechinus magellanicus (Larraín 1975, Guisado \& Castilla 1987, Gebauer \& Moreno 1995, Guisado 1995, Guisado et al. 1998, Vásquez 2001), pesquería (Stotz 2003, Moreno et al. 2006) y cultivo del erizo comestible (L. albus) (Bustos \& Olave 2001, Cárcamo et al. 2005). Antecedentes de la ecología larval y asentamiento de las especies registradas en Chile son escasos, reportándose la presencia de larvas en el plancton entre noviembre y febrero en la zona de Chiloé e islas Guiatecas (Bay-Schmith et al. 1981, Bustos et al. 1987, Kino \& Agatsuma 2007). Del asentamiento sólo se tienen antecedentes para $L$. albus en la costa chilena, observado principalmente en el intermareal rocoso entre diciembre y febrero, particularmente en grietas intermareales con abundante conchilla (Stotz et al. 1992).

Como en la mayoría de los invertebrados marinos bentónicos los equinodermos presentan un ciclo de vida complejo (McEdward \& Miner 2001), caracterizado por una etapa de desarrollo larval pelágica y una etapa adulta bentónica (Scheltema 1986, Fairweather 1991, Pineda 2000). La etapa planctónica se inicia con el desove de los adultos (Lawrence 2001), lo que en el caso de los erizos se ha descrito como un proceso sincrónico, influenciado por variables ambientales como la oferta de alimento para los adultos y el aumento de la temperatura del agua (Keats et al. 1984, Lamare \& Stewart 1998, Brewin et al. 2000, Lamare et al. 2002, Wing et al. 2003, Basch \& Tegner 2007, Kino \& Agatsuma 2007)

La distribución de las larvas en la columna de agua es modulada por variables físicas, químicas y biológicas del ambiente (Le Fèvre 1986, Fairweather 1991, Shanks 1995, Wing et al. 1995, Pineda 1999, Burdett-Coutts \& Metaxas 2004, Molinet et al. 2006), como por características propias de la especie (Scheltema 1986, Levin \& Bridges 1995, Young 1995, Sköld et al. 2003).

Con excepción de algunas larvas de peces y algunos estados larvales terminales de crustáceos, se ha establecido que la capacidad de natación horizontal de las larvas es de menor importancia en relación al transporte producido por diferentes procesos oceanográficos (Shanks 1995, Pineda 1999, Poulin et al. 2002, Molinet et al. 2006). De esta forma el transporte larval neto en equinodermos es conducido por procesos oceanográficos físicos y es afectado por la distribución vertical de las larvas en la columna de agua. La distribución vertical de las larvas es mediada por la respuesta de éstas a estímulos como luz y gravedad (Young 1995), alimento (Burdett-Coutts \& Metaxas 2004) y al efecto de gradientes ambientales verticales (e.g. salinidad). Estos últimos afectan la distribución del plancton, ya que representan barreras físicas o químicas para los organismos que intentan migrar a través de estos gradientes (Young 1995, Lougee et al. 2002, Gallager et al. 2004). Los gradientes salinos pueden ser importantes para la distribución del plancton en sistemas estratificados como los canales y fiordos del sur de Chile (Molinet 2005, Molinet et al. 2005, Landaeta \& Castro 2006, Molinet et al. 2008), ya que este sistema presenta una picnoclina bien definida (Pickard 1971, Silva et al. 1995), con flujos dominados por forzantes mareales, de viento y gradientes de densidad (Valle-Levinson et al. 2001, Cáceres et al. 2003, Cáceres \& Valle-Levinson 2004, Valle-Levinson \& Blanco 2004).

Por otro lado, eventos de asentamiento episódico han conducido a postular que varias fuentes de mortalidad afectan a las larvas, proponiéndose que la depredación sería la mayor fuente de mortalidad en estadíos tempranos (Thorson 1950, Morgan 1995) En Chile se ha reconocido que el asentamiento de invertebrados marinos puede ser fuertemente afectado por procesos oceanográficos de diferente escala espacial y temporal (Moreno et al. 1998, Marín \& Moreno 2002), mientras que el asentamiento de Echinoidea se ha descrito como espacial y temporalmente muy variable y dependiente de un conjunto de variables bióticas y abióticas, como la presencia de sustrato, la depredación, el estado nutritivo, etc. (Lawrence 2001).

El objetivo de este trabajo fue estudiar la variabilidad vertical y temporal (quincenal y diaria) de los estados tempranos planctónicos y post asentados de Echinoidea, durante el periodo primavera-verano en un canal austral 
del sur de Chile, y asociar la densidad de larvas, en el caso particular de Loxechinus albus, con el estado reproductivo de ejemplares adultos presentes en el área de estudio.

\section{Material y métodos}

Para cumplir con el objetivo de este estudio se realizaron i) recolecciones intensivas de plancton durante 24 horas cada 15 días en tres estratos de profundidad, ii) muestreo de post asentados en sustrato artificial y iii) monitoreo del índice gonadosomático de L. albus como referencia de la condición reproductiva de esta especie.

\section{Área de estudio}

El estudio se realizó en el extremo este del canal Lagreze $\left(43^{\circ} 54^{\prime} 30^{\prime \prime} \mathrm{S}, 73^{\circ} 15^{\prime} \mathrm{W}\right)$ ubicado en el noreste de Islas Guaitecas, Región de Aysén, Chile (Fig. 1). El área posee profundidades de hasta $63 \mathrm{~m}$ y presenta una columna de agua estratificada con salinidades de 27 a 33 ups, por sobre los $50 \mathrm{~m}$ de profundidad (Silva et al. 1995). En la zona aledaña al área de trabajo no existen datos de flujo, aunque la referencia de la carta SHOA N 717 indica flujos máximos de $1,5 \mathrm{~m} \mathrm{~s}^{-1}$ en mareas de sicigia. La recolección de muestras se desarrolló cercana a bancos de erizos (L. albus), informados en la pesquería del recurso en esa zona (Barahona et al. 2003) y en áreas con presencia de L. albus, P. magellanicus y A. dufresnei (C. Molinet, información no publicada, almacenada en registros de video submarino).

\section{Recolección y procesamiento de muestras de plancton}

En la zona este de Canal Lagreze se recolectaron tres muestras verticales de plancton a intervalos de $4 \mathrm{~h}(\mathrm{~N}=18$ muestras durante $24 \mathrm{~h})$, en tres estratos de profundidad: somero (0 a $10 \mathrm{~m})$, medio (10 a $30 \mathrm{~m})$ y profundo (30 a 50 m) Las muestras fueron recolectadas con una red cónica tipo WP-2 (40 cm de diámetro, $105 \mu \mathrm{m}$ de trama y sistema de cierre). Las muestras fueron preservadas en etanol al $70 \%$. El volumen filtrado fue estimado con la fórmula $V_{f}=a * h$, donde $a$ es el área de la boca de la red y $h$ es la distancia que recorre la red en el estrato respectivo.

En laboratorio, se separaron, contaron e identificaron todas las larvas de equinodermos utilizando un microscopio estereoscópico Olympus ${ }^{\circledR}$ con aumento 80x.

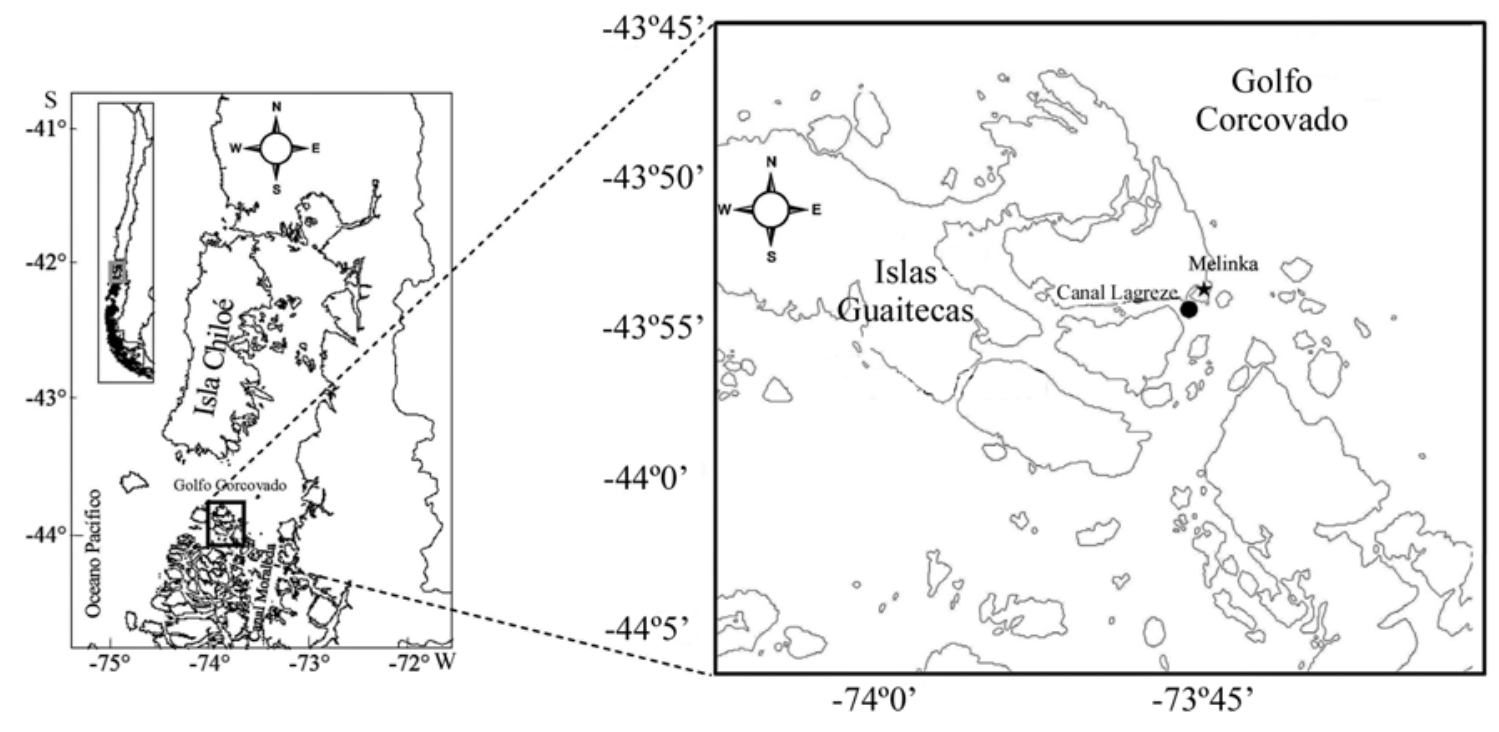

Figura 1

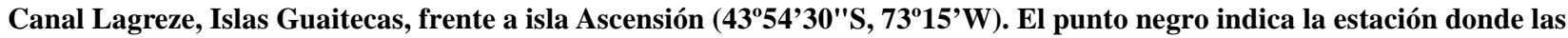
muestras de plancton fueron recolectadas, la estrella muestra la ubicación de Melinka, y el polígono gris muestra el área de recolección de erizos adultos y el área de instalación de colectores artificiales

Lagreze Channel, Guaitecas Islands, off Ascención island $\left(43^{\circ} 54^{\prime} 30^{\prime \prime} \mathrm{S}, 73^{\circ} 15^{\prime} \mathrm{W}\right)$. The black dot indicates the area where plankton samples were collected, the star indicates the location of Melinka, and the gray polygon, indicates sea urchin adults collection area and the area where artificial collestors were installed 


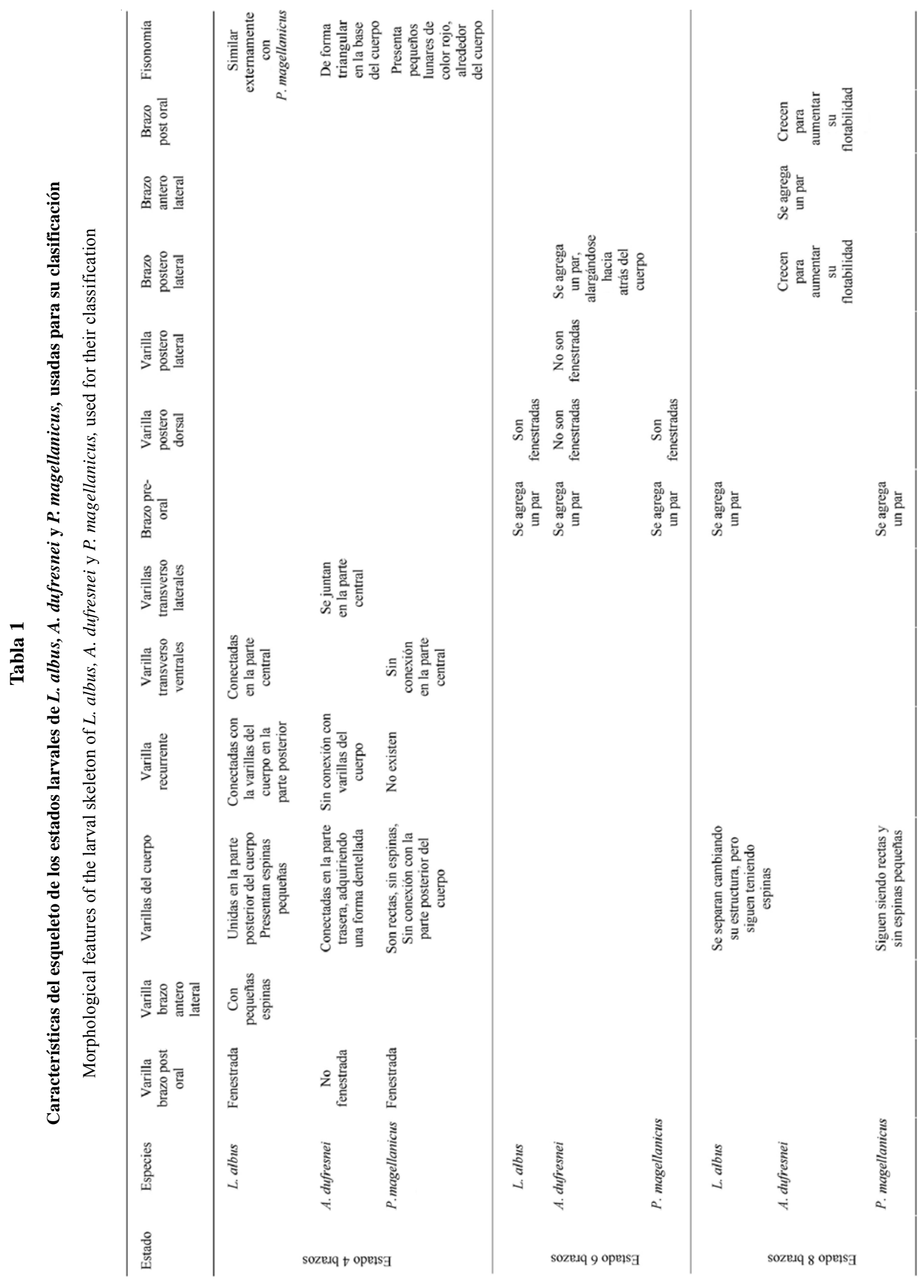


Para la identificación taxonómica de las larvas de las especies de Echinoidea L. albus, A. dufresnei y $P$. magellanicus y para la identificación de larvas de la clase Ophiuroidea se utilizaron los caracteres morfológicos descritos en la literatura científica (Arrau 1958, Geiger 1964, Emlet 1982, Bustos et al. 1987, Guisado \& Castilla 1987, Pennington \& Strathmann 1990, Guisado 1995, Bustos \& Olave 2001, Miner 2003, Primus 2005). Complementariamente se realizaron cultivos en laboratorio de larvas de erizo común Loxechinus albus y Pseudechinus magellanicus.

Las larvas fueron clasificadas en estados de desarrollo de acuerdo a la cantidad de brazos que poseían: cuatro, seis, ocho y diez brazos (Arrau 1958, Guisado 1995, Miner 2003, Cárcamo et al. 2005) (Tabla 1).

Simultáneamente, en cada muestreo se registró la temperatura y salinidad a cuatro profundidades de la columna de agua: $0,10,30$ y $50 \mathrm{~m}$ con un sensor electrónico multiparámetros $\mathrm{YSI}^{\circledR} 30$.

\section{Monitoreo de post asentados}

Para evaluar la relación entre la presencia de larvas en el plancton y de post asentados se utilizaron colectores artificiales. Estos fueron elaborados con tubos de PVC de $30 \mathrm{~cm}$ de largo y $10 \mathrm{~cm}$ de diámetro, cubiertos en ambos extremos por malla de $10 \mathrm{~mm}$ (adaptado de Harrold et al. (1991). Al interior del tubo se colocó $1 \mathrm{~m}$ de malla MIN JP (malla interna de colector para ostiones) enrollada como lo descrito por Barahona et al. (2003). Se instalaron 15 colectores en el área de estudio los que se mantuvieron desde octubre de 2005 hasta febrero de 2006, anclados al fondo en tres profundidades 1,15 y $40 \mathrm{~m}$ ( 5 colectores por profundidad). Los colectores fueron revisados mensualmente y la muestra preservada en etanol al 70\% para su posterior análisis en el laboratorio del Instituto de Fomento Pesquero (IFOP) de Putemún. Los colectores para los ejemplares asentados y el líquido fijador se vertieron sobre un tamiz de $350 \mu \mathrm{m}$ donde el colector fue lavado. Todo lo retenido en el tamiz fue traspasado a una placa y revisado bajo lupa. Los organismos pertenecientes a Echinoidea y Ophiuroidea (que fueron los que se observaron) fueron separados e identificados.

\section{Monitoreo de condición gonadal}

Con el fin de relacionar la variación del índice gonadosomático (IGS) con la presencia de larvas, se monitoreó mensualmente el IGS en ejemplares adultos de L. albus (usado como especie referencial) provenientes de bancos aledaños al área de recolección de muestras de plancton. Para tal efecto, se recolectaron 20 ejemplares por clase de talla (mayores a $40 \mathrm{~mm}$, talla de primera madurez sexual (Bay-Schmith et al. 1981) clasificados por rango de talla de $10 \mathrm{~mm}$, a los que se registró la longitud total, peso total, sexo de los individuos y peso de la gónada.

Para obtener el peso de las gónadas, éstas fueron extraídas removiendo la parte superior de la testa, y luego pesadas sobre una cápsula Petri, utilizando una balanza digital Pesamatic ${ }^{\circledR}$ de $0,01 \mathrm{~g}$ de precisión.

El sexo de cada individuo se determinó obteniendo un frotis de las mismas gónadas, el que se observó bajo microscopio con aumento de $200 \mathrm{X}$. La clasificación del sexo macho o hembra se realizó de acuerdo a lo descrito por Bustos \& Olave (2001).

\section{Análisis de datos}

Debido a que la densidad larval no mostró homogeneidad de varianza, se aplicó la prueba no paramétrica de Kruskal Wallis $(\mathrm{H})$ para evaluar en forma independiente el efecto de los muestreos, estratos y estados de desarrollo larval sobre la densidad de cada una de las especies y/o grupos de larvas identificadas. El efecto de la variable día-noche sobre la variación de la densidad fue evaluado utilizando la prueba Mann-Whitney (U) (Zar 1999).

Finalmente, se aplicó un análisis de correspondencia múltiple (ACM) (Legendre \& Legendre 1998) para evaluar la interacción entre los diferentes factores (especies, estados, estratos) y para la búsqueda de patrones dominantes que explicaran la densidad y distribución espacial y temporal de las especies de erizo recolectadas. Se ordenó la información en una tabla de contingencia, agrupando en categorías a los estados de desarrollo por número de brazos (4 categorías), mes del año en que se observó larvas (3 categorías), estratos de profundidad (3 categorías) y periodo día-noche (2 categorías) de las especies de Echinoidea identificadas (3).

El índice gonadosomático (IGS), para los ejemplares con tallas superiores o iguales a $40 \mathrm{~mm}$, se calculó mediante la fórmula:

$$
I G S=\frac{P T G H}{P T D} 100
$$

donde: PTGH es el peso total de la gónada húmeda y PTD es el peso total drenado del erizo (Lamare \& Stewart 1998).

El escaso registro de ejemplares post asentados y el restringido periodo de registro de larvas en la columna de agua no permitió explorar correlaciones entre ambas variables, por lo que sólo se presenta una descripción de estos resultados. 


\section{Resultados}

Durante el periodo de estudio se observó tanto en machos como hembras de L. albus una disminución gradual en el IGS, el que se inició con $10 \%$ en octubre de 2005 , disminuyó a aproximadamente $6 \%$ en enero de 2006 , e incremento levemente durante febrero de 2006 (Fig. 2). La proporción de machos durante 4 de los 5 meses de muestreo fue mayor que la proporción de hembras (Tabla 2).

Entre octubre de 2005 y febrero de 2006, se recolectó un total de 567 muestras en ocho expediciones de muestreo. La mayoría de las larvas encontradas fueron Ophiuroidea (88\%), las que se observaron de manera más frecuente en noviembre, principios de diciembre de 2005 y febrero de 2006, y sólo fue posible clasificar hasta el nivel de Clase. Larvas de Echinoidea fueron recolectadas sólo durante noviembre y principios de diciembre de 2005 en estados de 4 a 10 brazos, identificándose tres especies: Loxechinus albus (5\%), Arbacia dufresnei (6\%) y Pseudechinus magellanicus (0,3\%) (Tabla 1). Esta última especie se registró en sólo una muestra con dos larvas, por lo que no se aplicaron pruebas estadísticas para evaluar su variabilidad.

Tabla 2

Proporción de ejemplares macho y hembra de $L$. albus recolectados al este de Canal Lagreze, Islas Guaitecas durante el periodo de estudio

Proportion of L. albus male and female collected during the study period at the northeast of Lagreze Channel, Guaitecas Islands

\begin{tabular}{crrrrr}
\hline Sexo & Octubre & Noviembre & Diciembre & Enero & Febrero \\
\hline Macho & 57 & 53 & 57 & 54 & 50 \\
Hembra & 38 & 47 & 42 & 46 & 50 \\
Indeterminado & 5 & 0 & 1 & 0 & 0 \\
\hline
\end{tabular}

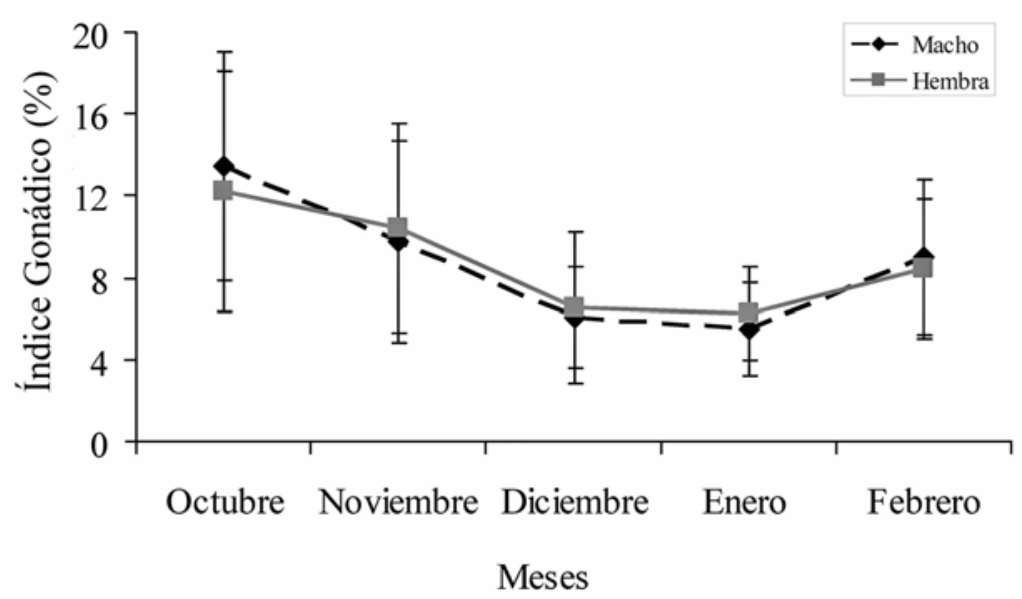

Figura 2

\section{Índice gonádico (IGS) de machos y hembras de Loxechinus albus recolectados en el área de estudio. Líneas de error muestran la desviación estándar del IGS para erizos machos y hembras}

Gonad index (GI) of male and female Loxechinus albus collected at the study area. Error lines show the standard deviation of IGS for male and female sea urchins 
Las mayores densidades promedio de larvas fueron observadas durante noviembre para todos los taxa. Ophiuridae ( 4 brazos $)=10,1$ larvas $\mathrm{m}^{-3}$, A. dufresnei $(6$ brazos $)=2,4$ larvas $\mathrm{m}^{-3} ;$ L albus $(8$ brazos $)=2,0$ larvas $\mathrm{m}^{-3}$; P. magellanicus ( 4 brazos $)=1,6$ larvas $\mathrm{m}^{-3}$ (Tabla $3)$, aunque los estados más frecuentes fueron los estados de 4 brazos para Echinoidea y de 6 brazos para Ophiuroidea.

La densidad larval estimada en diciembre fue significativamente menor a la densidad obtenida en noviembre en todas los taxa (Tabla 4). De aquellas, las larvas de Ophiuridae fueron nuevamente las más abundantes (2,2 larvas $\mathrm{m}^{-3}$ en estado de 8 brazos), seguidas por larvas de $L$. albus $\left(1,2\right.$ larvas $\left.\mathrm{m}^{-3}\right)$ y $A$. dufresnei $\left(0,6\right.$ larvas $\left.\mathrm{m}^{-3}\right)$, estas últimas en estado de cuatro brazos. En febrero sólo se observaron larvas de Ophiuridae en baja densidad $\left(0,8\right.$ larvas $\mathrm{m}^{-3}$ en estado de 6 brazos) (Tabla 3 ).

Tabla 3

Densidad promedio de los diferentes estados larvales de Echinoidea desde octubre 2005 a febrero 2006 en el canal Lagreze, sur de Chile. Promedio de densidad (larvas $\mathrm{m}^{-3}$ ) y desviación estándar en paréntesis

Mean density of larval stages of Echinoidea from October 2005 to February 2006 at Lagreze Channel, southern Chile. Mean density (larvae $\mathrm{m}^{-3}$ ) and standard deviation in parenthesis

\begin{tabular}{|c|c|c|c|c|c|c|c|c|c|}
\hline \multirow[b]{2}{*}{ Especie } & \multirow[b]{2}{*}{$\mathrm{N}^{\circ}$ de brazos } & \multicolumn{4}{|c|}{2005} & \multicolumn{4}{|c|}{2006} \\
\hline & & 31-Oct & 18-Nov & 04-Dic & 18-Dic & 06-Ene & 18-Ene & 09-Feb & 20-Feb \\
\hline \multirow[t]{3}{*}{ Arbacia dufresnei } & 4 & 0 & $1,8(1,22)$ & $0,6(0,2)$ & 0 & 0 & 0 & 0 & 0 \\
\hline & 6 & 0 & $2,4(1,3)$ & 0 & 0 & 0 & 0 & 0 & 0 \\
\hline & 10 & 0 & 0 & 0,4 & 0 & 0 & 0 & 0 & 0 \\
\hline \multirow[t]{3}{*}{ Loxechinus albus } & 4 & 0 & $1,7(1,3)$ & $1,2(0,6)$ & 0 & 0 & 0 & 0 & 0 \\
\hline & 6 & 0 & $1,1(0,6)$ & 0 & 0 & 0 & 0 & 0 & 0 \\
\hline & 8 & 0 & $2,0(0,6)$ & 0 & 0 & 0 & 0 & 0 & 0 \\
\hline \multicolumn{10}{|l|}{ Pseudechimus } \\
\hline \multirow[t]{3}{*}{ magellanicus } & 4 & 0 & 1,6 & 0 & 0 & 0 & 0 & 0 & 0 \\
\hline & 6 & 0 & & 0 & 0 & 0 & 0 & 0 & 0 \\
\hline & 8 & 0 & & 0 & 0 & 0 & 0 & 0 & 0 \\
\hline \multirow[t]{3}{*}{ Ophiopluteus } & 4 & 0 & $10,1(7,2)$ & $1,5(0,4)$ & 0 & 0 & 0 & 0 & 0 \\
\hline & 6 & 0 & $9,8(6,6)$ & $1,3(0,6)$ & 0 & 0 & 0 & 0 & 0,8 \\
\hline & 8 & 0 & $9,2(6,6)$ & $2,2(1,6)$ & 0 & 0 & 0 & 0 & 0,4 \\
\hline
\end{tabular}

Tabla 4

Resumen de la prueba de Kruskal-Wallis (H) y Mann-Whitney (U) para evaluar el efecto del estado de desarrollo, estrato de profundidad, periodo de muestreo y periodo del día sobre la densidad de las diferentes especies de Echinoidea. Resultados de pruebas estadísticas significativas $(P<0,05)$ se muestran en negrita

Summary of values of Kruskal-Wallis test (H) and Mann-Whitney test (U) to evaluate the effect of developmental stage, sampling stratum, sampling period and diel period on larval density of different species of Echinoidea. Result of

significant statistical tests $(P<0.05)$ are shown in bold

\begin{tabular}{|c|c|c|c|}
\hline Factor & A. dufresnei & L. albus & Ophiuroideos \\
\hline Muestreo & $\mathbf{H}_{0,05,2,148}=22 ; P<0,05$ & $\mathrm{H}_{0.05,2,151}=26,3 ; P<0,05$ & $\mathrm{H}_{0,05,3,295}=51,2 ; P<0,05$ \\
\hline Estrato de profundidad & $\mathrm{H}_{0,05,2,148}=9,2 ; P<0,05$ & $\mathrm{H}_{0.05,2,151}=25,7 ; P<0,05$ & $\mathrm{H}_{0,05,3,295}=32,5 ; P<0,05$ \\
\hline Periodo (día-noche) & $\mathrm{U}_{0,05,53,70}=1621 ; P>0,05$ & $\mathrm{U}_{0,05,70,69}=1927 ; P<0,05$ & $\mathrm{U}_{0,05,103,150}=6876 ; P>0,05$ \\
\hline Estado ( $\mathrm{N}^{\circ}$ de brazos) & $\mathrm{H}_{0,05,2,148}=34,8, P<0,05$ & $\mathrm{H}_{0.05,2,151}=10.5, P<0,05$ & $\mathrm{H}_{0,05,3,295}=5,3, P>0,05$ \\
\hline
\end{tabular}



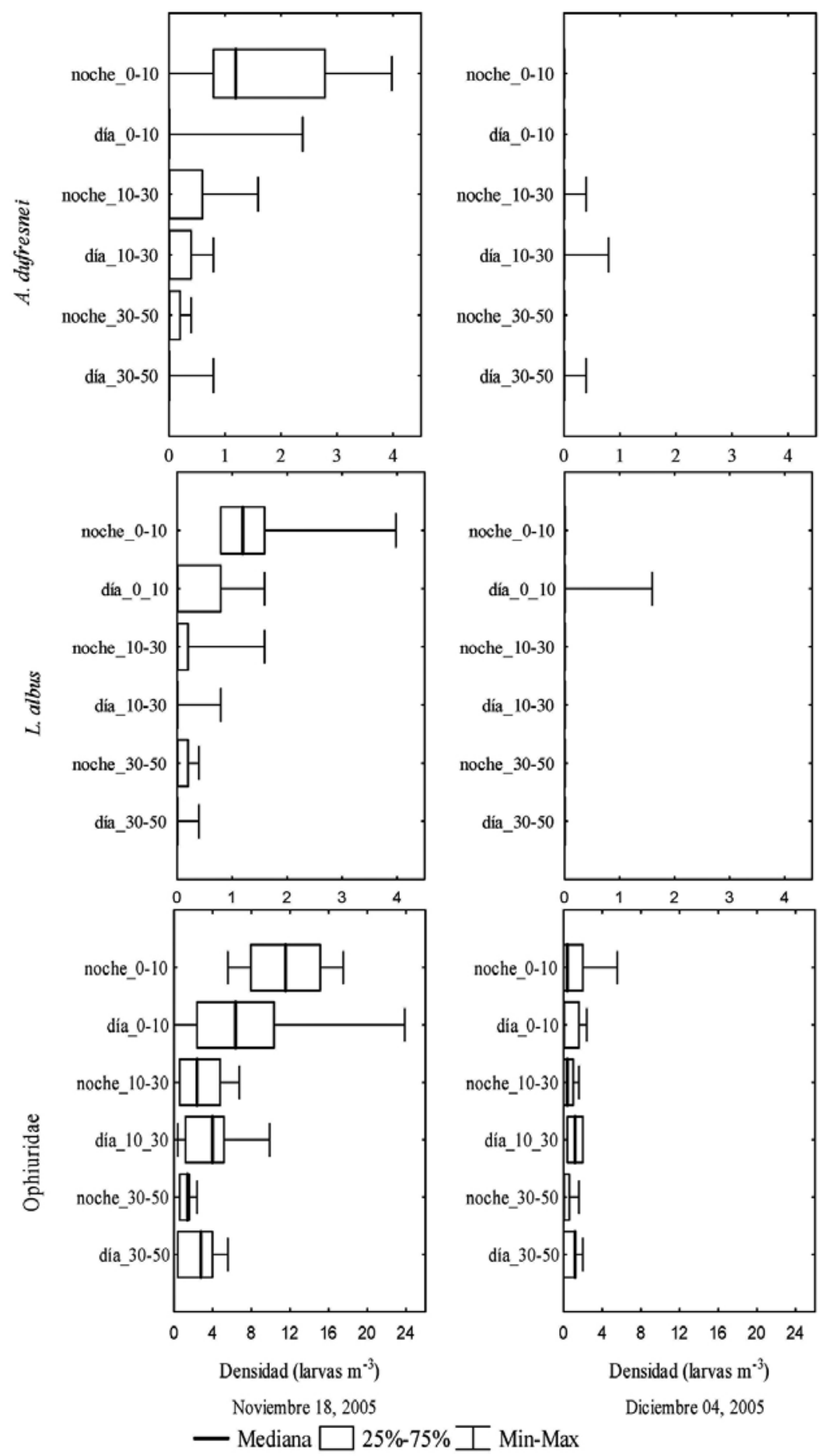

Figura 3

Distribución vertical de larvas de equinodermos durante el día y la noche en canal Lagreze. Los estratos de muestreo vertical en metros y la densidad larval en individuos por $\mathbf{~ m}^{3}$

Vertical distribution of echinoderm larvae, collected during day and night times at Lagreze Channel. Vertical sampling strata in meters and larval density in individuals per $\mathrm{m}^{3}$ 

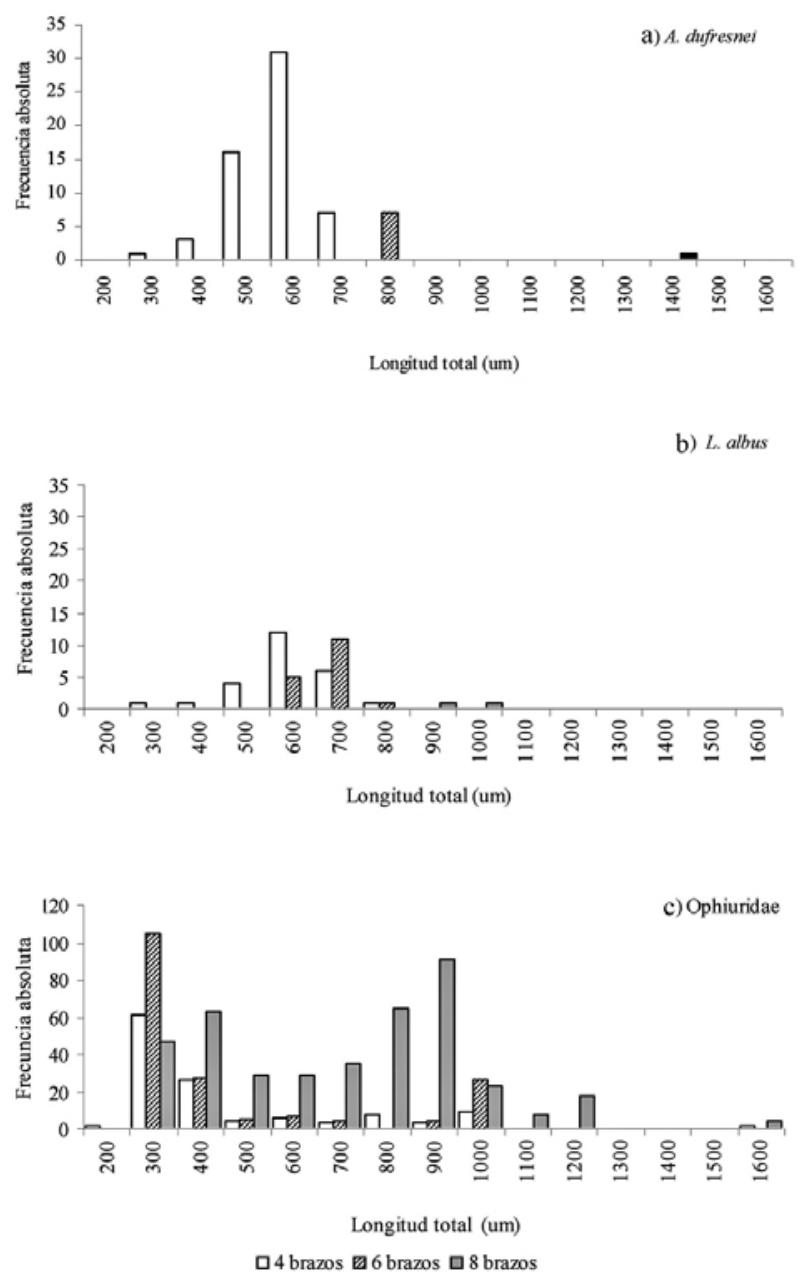

Figura 4

Distribución de tamaño larval (largo del brazo, en ìm) según estado de desarrollo (número de brazos) de a) Ophiopluteus, b) Loxechinus albus y c) Arbacia dufresnei, recolectadas desde noviembre de 2005 a febrero de 2006 en canal Lagreze

Larval size distribution (arm length, in ì $\mathrm{m}$ ) and developmental stage (number of arms) of a) Ophiopluteus, b) Loxechinus albus and c) Arbacia dufresnei, collected from November 2005 to February 2006 at Lagreze Channel

La densidad de larvas de A. dufresnei, L. albus y Ophiuridae varió significativamente entre los estratos de profundidad $(P<0,05)$, observándose un gradiente de mayor a menor densidad desde superficie a fondo (Fig. 3 ). Además se observó que sólo la densidad larval de $L$. albus varió significativamente entre el día y la noche (U: 1927; $P<0,05)$ con mayor densidad de larvas durante la noche (mediana noche $=1,2$ larvas $\mathrm{m}^{-3}$ vs. mediana día $=0$ larvas $\mathrm{m}^{-3}$, respectivamente) (Tabla 4, Fig. 3).
Tabla 5

\section{Post asentados de Echinoidea observados en colectores instalados en el área de estudio en tres estratos de profundidad. (L.a.= Loxechinus albus, Op= Ophiuroidea)}

Post-settlers of Echinoidea obtained in the collectors installed in the study area at three depths (L.a.= Loxechinus albus, $\mathrm{Op}=$ Ophiuroidea)

\begin{tabular}{llcc}
\hline \multicolumn{1}{c}{ Mes } & \multicolumn{3}{c}{ Estrato de profundidad } \\
\hline & $1 \mathrm{~m}$ & $15 \mathrm{~m}$ & $40 \mathrm{~m}$ \\
Noviembre & 0 & 0 & 0 \\
Diciembre & 1 L.a. & 3 L.a. & 4 L.a. \\
& 1 Op. & & \\
Enero & 0 & 0 & 0 \\
Febrero & 0 & 1 L.a. & 0 \\
\hline
\end{tabular}

La densidad de $A$. dufresnei fuer mayor en los estados de 4 y 6 brazos $(P<0,05)$ (Tablas 3 y 4, Fig. 4 a,b). Para L. albus, los estados 4 y 6 brazos fueron más frecuentes, los que se observaron de manera más homogénea durante el periodo en que estuvieron presentes, y en más baja densidades que el estado 8 brazos. Los diferentes estados larvales de Ophiuroidea no presentaron diferencias significativas en su densidad $(P>0,05)$ (Fig. 4c, Tablas 3 y 4$)$.

El análisis de correspondencia múltiple (ACM) mostró que las dos primeras dimensiones explican significativamente el 17 y $12 \%$ de la variabilidad de la densidad de las larvas (Fig. 5). Se observó una asociación en la densidad larval de L. albus 6 y 8 brazos con el factor superficie y el factor noche, mientras que larvas de $A$. dufresnei 4 brazos se asociaron al factor estrato profundo y medio y al factor día. La densidad de larvas de $P$. magellanicus 4 brazos y L. albus 8 brazos fueron bajas, ambas registradas durante un periodo de muestreo y en una muestra, por lo que aparecen representadas en un extremo del gráfico de ordenación. Similar patrón fue observado para la densidad larval durante diciembre en la segunda dimensión del gráfico de ordenación.

El número de post asentados observado fue escaso, con un total de 8 ejemplares de L. albus (en diciembre y febrero) y 5 Ophiuroidea (exclusivamente en diciembre), distribuidos en los tres estratos de profundidad analizados (Tabla 5).

Durante el periodo de muestreo, la columna de agua estudiada en el canal Lagreze no mostró gradientes verticales importantes en la temperatura y salinidad (entre superficie y $50 \mathrm{~m}$ de profundidad). Tampoco se 


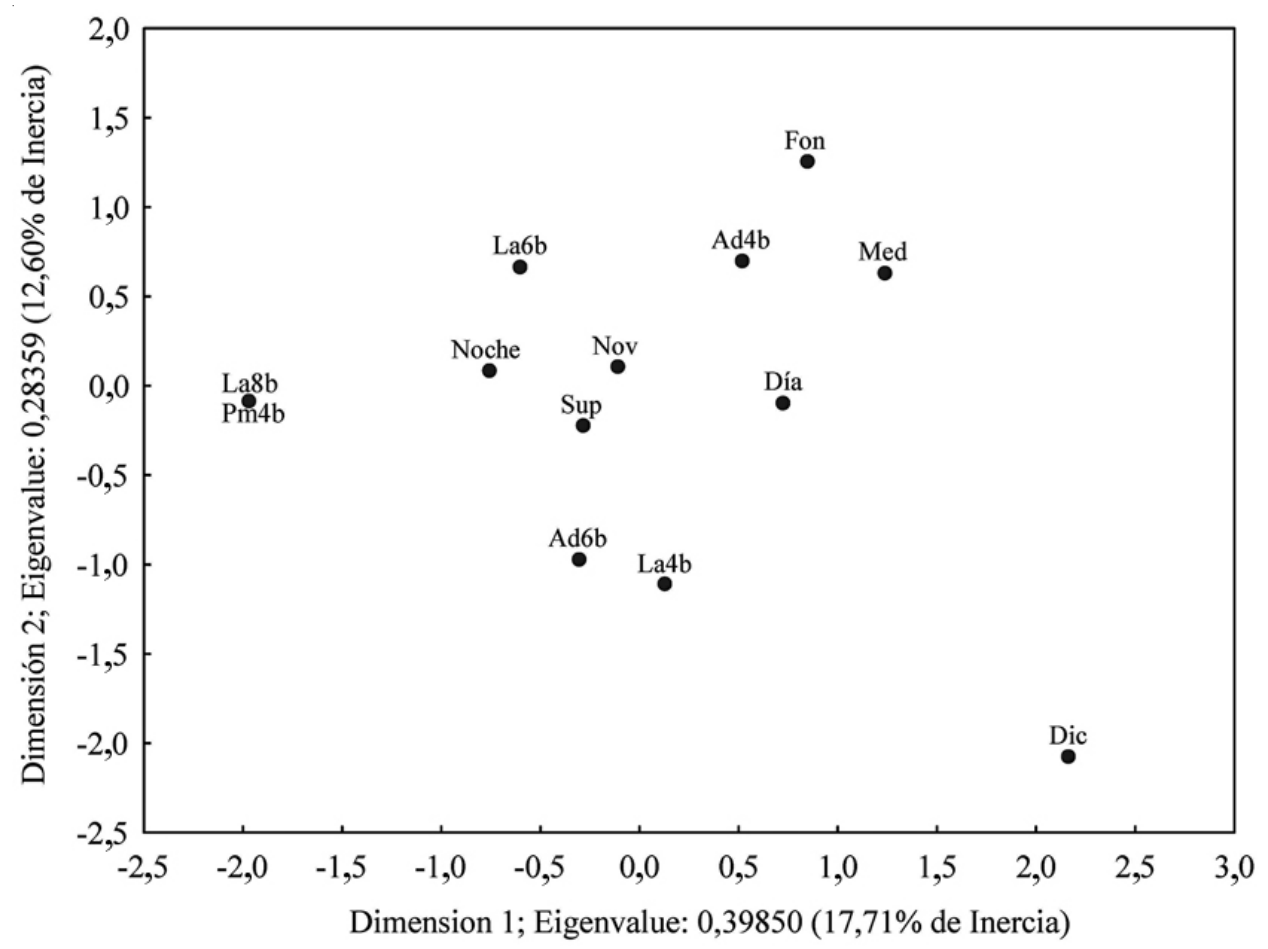

Figura 5

Diagrama de ordenación del análisis de correspondencia múltiple. La4b, L. albus en estado 4 brazos; La6b, L. albus en estado 6 brazos; La8b, L. albus en estado 8 brazos; Ad4b, A. dufresnei en estado 4 brazos; Ad6b, A. dufresnei en estado 6 brazos; Pm4b, P. magellanicus en estado 4 brazos; Sup., estrato superficial; Med., estrato medio; Fon., estrato fondo; Nov., Noviembre; Dic., Diciembre; Noche; Día

Ordination plot of multiple correspondence analysis. La4b, L. albus; Ad, A. dufresnei; Pm, P. magellanicus; Sup., upper; Med., middle; Fon., deep; Nov., November; Dic., December; Night; Day; 4br, 6br and 8 br developmental stage 4, 6 and 8 arms

a) Salinidad

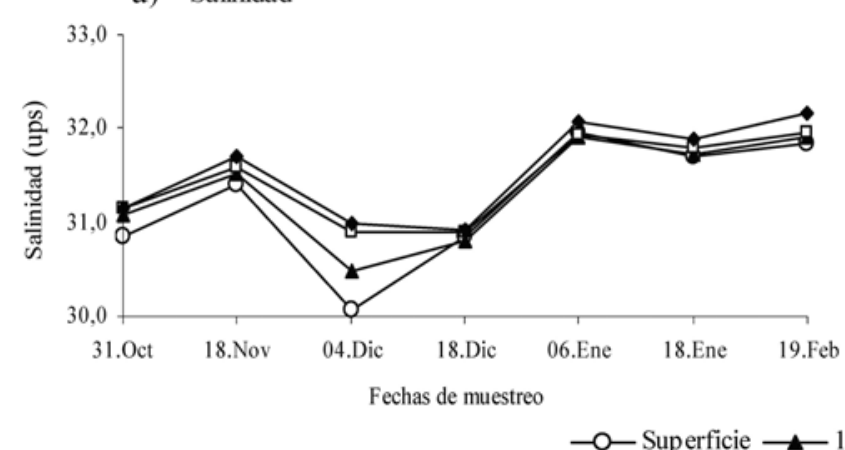

b) Temperatura

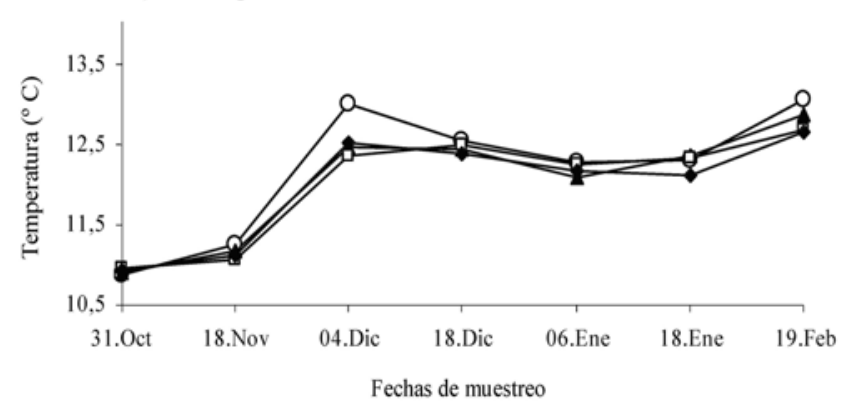

Figura 6

Variabilidad espacial (vertical) y temporal (quincenal) de a) salinidad (ups) y b) temperatura ( ${ }^{\circ} \mathrm{C}$ ) en el sitio de muestreo

Spatial (vertical) and temporal (bi-weekly) variability of a) salinity (psu), and b) temperature $\left({ }^{\circ} \mathrm{C}\right)$ at the sampling area 
observaron variaciones temporales considerables entre octubre de 2005 y febrero de 2006 para ambas variables monitoreadas. Solo a inicios de diciembre se observó una leve variación salina y térmica entre los estratos, fluctuando entre 30 y 31 ups y $13^{\circ} \mathrm{C}$ y $\sim 12^{\circ} \mathrm{C}$ entre superficie y $50 \mathrm{~m}$ de profundidad, respectivamente. La salinidad varió durante el periodo de estudio entre 30 a 32 ups, durante diciembre y febrero respectivamente y la temperatura entre $11^{\circ} \mathrm{C}$ y $13^{\circ} \mathrm{C}$, entre octubre y febrero, respectivamente (Fig. 6).

\section{Discusión}

Los resultados obtenidos en este estudio constituyen las primeras observaciones sobre distribución vertical de estados tempranos de Echinoidea en ambiente natural en un canal del sur de Chile. Estos resultados muestran evidencia de un restringido periodo de presencia de larvas de equinodermos en la columna de agua, ya que sólo fueron recolectadas en relativa abundancia durante un muestreo en noviembre, pese al intenso esfuerzo de muestreo desplegado ( 7 muestras diarias, en tres estratos de profundidad, con sus réplicas, cada 15 días, con un total de 567 muestras).

En el caso particular de L. albus, el IGS siguió un patrón similar a lo reportado entre $42^{\circ}$ y $45^{\circ} \mathrm{S}$ (BaySchmith et al. 1981, Zamora \& Stotz 1992, Arias et al. 1995, Barahona et al. 2003). La disminución del IGS en $L$. albus coincidió con el periodo de mayor frecuencia de larvas en el plancton, aproximadamente 15 días antes del registro de post asentados en los colectores artificiales. Esto sugiere que para el caso particular de este estudio el periodo reproductivo de $L$. albus se extendió principalmente entre noviembre y diciembre de 2005, lo que coincide por lo propuesto por Bay-Schmith et al. (1981) y Arias et al. (1995).

Para A. dufresnei y P. magellanicus se ha reportado que las mayores densidades larvales ocurren entre julio y marzo, y particularmente en agosto $\left(\sim 63\right.$ larvas $\mathrm{m}^{-3}$ de A. dufresnei y $\sim 127$ larvas $\mathrm{m}^{-3}$ de $P$. magellanicus, en estado de cuatro brazos) en el mar interior de Chiloé (Bustos et al. 1987). Si el periodo de desove ocurre principalmente durante otoño-invierno para A. dufresnei y $P$. magellanicus, entonces este patrón temporal podría explicar las bajas densidades larvales de estas especies durante el presente trabajo.

Por otro lado, entre 1987 y 1989 Kino \& Agatsuma (2007) reportaron densidades larvales promedio de $L$. albus entre 4 y 18 larvas de 4 brazos $\mathrm{m}^{-3}$ en Queilen $\left(42^{\circ} 50^{\prime} \mathrm{S}\right)$ y Quellón $\left(43^{\circ} 05^{\prime} \mathrm{S}\right)$, respectivamente, con máximo de hasta 72 larvas de 4 brazos $\mathrm{m}^{-3}$, mientras que prácticamente no se registraron larvas en Hueihue
(42³0'S). En el estudio antes mencionado la densidad larval varió interanualmente y el periodo de presencia de larvas se extendió por al menos cuatro meses entre noviembre y marzo de cada año, mientras que en nuestro estudio el periodo de presencia de larvas de $L$ albus se restringió prácticamente a un mes. Entre noviembre de 1980 y marzo de 1981 Bay-Schmith et al. (1981) reportaron larvas de Ophiuroidea, L. albus y Arbacia sp., en proporciones similares a las encontradas en este estudio, durante noviembre y enero, con L. albus registrado sólo durante diciembre. Aunque el estudio incluyó muestreos en Islas Guaitecas, lamentablemente la información registrada fue sólo cualitativa y no permite compararla con nuestros resultados.

La duración de los estados larvales de A. dufresnei y P. magellanicus no ha sido descrita en la literatura, mientras que para L. albus, Bustos \& Olave (2001) han reportado un tiempo de desarrollo larval de 22 días en laboratorio a $18^{\circ} \mathrm{C}$ entre el desove y el estado precompetente. En el área de estudio se registraron temperaturas entre 11 y $13^{\circ} \mathrm{C}$, por lo que es probable un periodo de desarrollo larval más prolongado para L. albus.

Por otra parte, larvas Ophiuroidea fueron registradas durante un periodo de tiempo más extenso (noviembre, diciembre y febrero). Probablemente, estas larvas pertenecen a varias especies de Ophiuroidea que habitan en el área de estudio y que sólo fueron identificadas a nivel de Clase debido que no se encontró literatura disponible acerca de ciclos reproductivos de este grupo en Chile.

El área de estudio corresponde a zonas con relativa abundancia de adultos de L. albus, A. dufresnei y $P$. magellanicus (C. Molinet, información no publicada), por lo que se esperaba recolectar una mayor abundancia de larvas, al menos de L. albus (ya que las otras dos especies no tendrían actividad reproductiva en esta época). La baja densidad larval observada en este estudio coincidió con un bajo número de post asentados de L. albus (observado en sustratos artificiales). Este restringido periodo de presencia de larvas de L. albus en el ambiente, la baja densidad larval y, los pocos post asentados, aunque localizado a un área en este estudio, podría corresponder a variabilidad anual descrita para Echinoidea (Lawrence 2001) o también estar asociado a los efectos de la intensa pesquería que se ha desarrollado en la zona de estudio en los últimos 20 años (Zuleta et al. 2008) y debería ser estudiado a través de un número mayor de estaciones de muestreo, a través de un programa de monitoreo de largo alcance. 
En los canales del sur de Chile la circulación costera es dinámica (Valle Levinson et al. 2001, Cáceres et al. 2003, Valle-Levinson \& Blanco 2004), por lo que no se puede descartar que las larvas fueron dispersadas por procesos advectivos hacia otras áreas, como ha sido observado para larvas de Strongylocentrotus purpuratus (Stimpson, 1857) y Evechinus chloroticus (McRae, 1959) (Botsford 2001, Wing et al. 2003) en otros sistemas de fiordos y canales. Futuros estudios debieran considerar una mayor cobertura espacial horizontal en el muestreo.

En este estudio se recolectó un mayor número de larvas de Echinoidea durante la noche y en superficie (significativo para el caso de las larvas de L. albus), mientras que durante el día las larvas fueron observadas de manera más homogénea en la columna de agua. Esto podría estar relacionado a una conducta migratoria nictimeral asociada a un comportamiento alimenticio como lo observado para los erizos Lytechinus variegatus (Lamarck, 1816) y Strongylocentrotus droebachiensis (Mueller, 1776) (Burdett-Coutts \& Metaxas 2004). La variabilidad en la distribución vertical ha sido observada para otras larvas de invertebrados marinos en el mundo (Young 1995) y particularmente en al área de estudio para larvas del gasterópodo Concholepas concholepas (Bruguière, 1789) (Molinet et al. 2008) y el langostino Munida gregaria (Fabricius, 1793) (León et al. 2008). Estos dos últimos casos, asociados a interacciones con variables físicas y químicas de la columna de agua y al efecto día-noche. En este estudio la temperatura y salinidad no parecieron tener un efecto claro sobre la abundancia de larvas, probablemente debido al corto periodo en que estas fueron registradas en el plancton, por lo que muestreos más intensivos o estudios en laboratorio debieran avanzar en esta línea.

Adicionalmente, se ha observado que la presencia de depredadores puede generar conductas de evasión en larvas echinoplutei de $S$. droebachiensis que afectan su distribución vertical (Metaxas \& Burdett-Coutts 2006). Estos mecanismos de respuesta a la concentración de alimento, depredadores y a gradientes verticales en la estructura de la columna de agua deben ser estudiados en futuros trabajos ya que pueden tener efectos significativos en la densidad larval y por lo tanto en la conectividad de las poblaciones adultas de organismos meroplanctónicos como ha sido propuesto por Landaeta \& Castro (2006) y para larvas de peces.

\section{Agradecimientos}

Se agradece la valiosa colaboración de los muestreadores de campo, el Sr. Víctor Ruiz y Srta. Andrea Ruiz. Esta investigación fue financiada a través del Proyecto FIP
2003-13 "Bases biológicas para rotación de áreas en el recurso erizo (fase 2)". Esta publicacion fue financiada por la Universidad Austral de Chile. Tres evaluadores anónimos contribuyeron a mejorar sustancialmente el contenido del presente trabajo.

\section{Literatura citada}

Arias E, N Barahona, G Jerez \& E Lozada. 1995. Monitoreo del recurso erizo en la X y XI región. Informe Final FIP 93-13: 1-74.

Arrau L. 1958. Desarrollo del erizo comestible en Chile, Loxechinus albus (Molina). Revista de Biología Marina 7: 39-61.

Barahona N, JM Orensanz, AM Parma, G Jerez, C Romero, H Miranda, A Zuleta, V Catasti \& P Galvez. 2003. Bases biológicas para la rotación de áreas en el recurso erizo. Informe Final. Proyecto FIP 2000-18: 1-200.

Basch L \& MJ Tegner. 2007. Reproductive responses of Purple sea urchin (Strongylocentrotus purpuratus) populations to environmental conditions across a coastal depth gradient. Bulletin of Marine Science 81: 255-282.

Bay-Schmith E, C Werlinger \& J Silva. 1981. Ciclo anual de reproducción del recurso erizo Loxechinus albus entre la X y XII Región, 68 pp. Universidad de Concepción, Concepción.

Botsford LW. 2001. Physical influences on recruitment to California Current invertebrates population on multiples scales. Journal of Marine Science 58: 1081-1091.

Brewin PE, MD Lamare, JA Keogh \& PV Mladenov. 2000. Reproductive variability over a four-year period in the sea urchin Evechinus chloroticus (Echinoidea : Echinodermata) from differing habitats in New Zealand. Marine Biology 137: 543-557.

Burdett-Coutts V \& A Metaxas. 2004. The effect of the quality of food patches on larval vertical distribution of the sea urchins Lytechinus variegatus (Lamarck) and Strongylocentrotus droebachiensis (Mueller). Journal of Experimental Marine Biology and Ecology 308: 221-236.

Bustos E \& S Olave. 2001. El cultivo del erizo (Loxechinus albus), 23 pp. Instituto de Fomento Pesquero, Puerto Montt.

Bustos H, R Troncoso, J Valencia \& A Reyes. 1987. Repoblación y cultivo de la ostra chilena y del erizo en la isla de Chiloé, 138 pp. Instituto de Fomento Pesquero, Puerto Montt.

Cáceres M \& A Valle-Levinson. 2004. Transverse variability of flow on both sides of a sill/contraction combination in a fjord-like inlet of southern Chile. Estuarine, Coastal and Shelf Science 60: 325-338.

Cáceres M, A Valle-Levinson, J Fierro, M Bello \& M Castillo. 2003. Variabilidad longitudinal del flujo en canales con influencia batimétrica y topográfica. En: CONA (ed). Resultados Crucero Cimar 8 Fiordos, Informes Preliminares, pp. 17-24. Comité Oceanográfico Nacional, Valparaíso. 
Cárcamo PF, AL Candia \& OR Chaparro. 2005. Larval development and metamorphosis in the sea urchin Loxechinus albus (Echinodermata: Echinoidea): Effects of diet type and feeding frequency. Aquaculture 249: 375-386.

Emlet RB. 1982. Echinoderm calcite: A mechanical analysis from larval spicules. The Biological Bulletin 163: 264-275.

Fairweather PG. 1991. Implications of "supply-side ecology" for environmental assessment and management. Trens in Ecology of Evolution 31: 60-63.

Gallager SM, H Yamazaki \& CS Davis. 2004. Contribution of fine-scale vertical structure and swimming behavior to formation of plankton layers on Georges Bank. Marine Ecology Progress Series 267: 27-43.

Gebauer P \& CA Moreno. 1995. Experimental validation of the growth rings of Loxechinus albus (Molina, 1872) in the southern Chile (Echinodermata: Echinoidea). Fisheries Research 21: 423-435.

Geiger SR. 1964. Echinodermata: Larvae. Classes: Ophiuroidea and Echinoidea (Plutei). Conseil International pour L'exploration de la Mer, Fiches d'identification du Zooplancton 105: 1-4.

Guisado C. 1995. Estrategias de desarrollo larval y ciclo de vida en dos especies de echinoideos regulares del sur de Chile. Tesis de Magíster, Facultad de Ciencias, Universidad Austral de Chile, Valdivia, 89 pp.

Guisado C \& JC Castilla. 1987. Historia de vida, reproducción y avances en el cultivo del erizo comestible chileno Loxechinus albus (Molina, 1782) (Echinoidea: Echinidae). En: Arana P (ed). Manejo y desarrollo pesquero, pp. 5968. Editorial Universitaria, Valparaíso.

Guisado C, E Arias \& E Peréz. 1998. Estudio reproductivo del erizo en las regiones I-VIII. Informe Final. Proyecto FIP-IT/96-44: 1-233.

Harrold C, S Lisin, KH Light \& S Tudor. 1991. Isolating settlement from recruitment of sea urchins. Journal of Experimental Marine Biology and Ecology 147: 81-94.

Keats DW, DH Steele \& GR South. 1984. Depth-dependent reproductive output of the green sea urchin, Strongylocentrotus droebachiensis (O.F. Müller), in relation to the nature and availability of food. Journal of Experimental Marine Biology and Ecology 80: 77-91.

Kino S \& Y Agatsuma. 2007. Reproduction of sea urchin Loxechinus albus in Chiloé Island, Chile. Fisheries Science 73: $1265-1273$.

Lamare MD \& BG Stewart. 1998. Mass spawning by the sea urchin Evechinus chloroticus (Echinodermata : Echinoidea) in a New Zealand fjord. Marine Biology 132: 135-140.

Lamare MD, PE Brewin \& MF Barker. 2002. Reproduction of the sea urchin Evechinus chloroticus (Echinodermata: Echinoidea) in a New Zealand fjord. New Zealand Journal of Marine and Freshwater Research 36: 719-732.
Landaeta MF \& L Castro. 2006. Larval distribution and growth of the rockfish, Sebastes capensis (Sebastidae, Pisces), in the fjords of southern Chile. ICES Journal of Marine Science 63: 714-724.

Larraín AP. 1975. Los equinoideos regulares fósiles y recientes de Chile. Gayana Zoología 35: 1-188.

Lawrence JM. 2001. Edible sea urchins: Biology and ecology, 419 pp. Elsevier, Tampa.

Le Fèvre J. 1986. Aspects of the biology of frontal systems. Advances in Marine Biology 23: 163-299.

Legendre P \& L Legendre. 1998. Numerical ecology, 853 pp. Elsevier, Amsterdam.

León R, LR Castro \& M Cáceres. 2008. Dispersal of Munida gregaria (Decapoda: Galatheidae) larvae in Patagonian channels of southern Chile. ICES Journal of Marine Science 65(7): 1131-1143

Levin LA \& TS Bridges. 1995. Pattern and diversity in reproduction and development. En: McEdward L (ed). Ecology of marine invertebrate larvae, pp. 1-48. CRC Press, Boca Raton.

Lougee LA, SM Bollens \& SR Avent. 2002. The effects of haloclines on the vertical distribution and migration of zooplankton. Journal of Experimental Marine Biology and Ecology 278: 111-134.

Marín V \& CA Moreno. 2002. Wind driven circulation and dispersal: A review of its consequences in coastal benthic recruitment. En: Castilla JC \& JL Largier (eds). The oceanography and ecology of the nearshore and bays in Chile, pp. 47-63. Ediciones Universidad Católica de Chile, Santiago.

McBride SC, RJ Price, PD Tom, JM Lawrence \& AL Lawrence. 2004. Comparison of gonad quality factors: color, hardness and resilience, of Strongylocentrotus franciscanus between sea urchins fed prepared feed or algal diets and sea urchins harvested from the Northern California fishery. Aquaculture 233: 405-422.

McEdward L \& BG Miner. 2001. Echinoid larval ecology. En: Lawrence JM (ed). Edible sea urchins: Biology and ecology 32: 59-78. Elsevier, Tampa.

Metaxas A \& V Burdett-Coutts. 2006. Response of invertebrate larvae to the presence of the ctenophore Bolinopsis infundibulum, a potential predator Journal of Experimental Marine Biology and Ecology 334: 187-195.

Miner BG. 2003. Evolution of phenotypic plasticity: Insights from Echinoid larvae, 94 pp. University of Florida, Gainesville.

Molinet C. 2005. Spatial and temporal distribution of Concholepas concholepas (Gastropoda, Muricidae) veliger larvae in the inland seas of northwest Patagonian. PhD Dissertation, Instituto de Ecología y Evolución. Universidad Austral de Chile, Valdivia, 124 pp. 
Molinet C, A Arevalo, MT Gonzalez, CA Moreno, J Arata \& E Niklitschek. 2005. Patterns of larval distribution and settlement of Concholepas concholepas (Bruguiere, 1789) (Gastropoda, Muricidae) in fjords and channels of southern Chile. Revista Chilena de Historia Natural 78: 409-423.

Molinet C, A Valle-Levinson, CA Moreno, M Cáceres, M Bello \& M Castillo. 2006. Effects of sill processes on the distribution of epineustonic competent larvae in a stratified system of Southern Chile. Marine Ecology Progress Series 324: $95-104$

Molinet C, E Niklitschek, CA Moreno \& A Arevalo. 2008. Vertical distribution of early and competent larvae of Concholepas concholepas in two systems of Chilean inland seas. Marine Biology 153: 779-787.

Moreno CA, G Asencio, W Duarte \& V Marín. 1998. Settlement of the muricid Concholepas concholepas and its relationship with El Niño and coastal upwellings in southern Chile. Marine Ecology Progress Series 167: 171175 .

Moreno CA, N Barahona, C Molinet, JML Orensanz, AM Parma \& A Zuleta. 2006. From crisis to institutional sustainability in the Chilean sea urchin fishery. En: McClanahan T\& JC Castilla (eds). Fisheries management: progress towards sustainability, pp. 43-67. Blackwell Publishing, Mombasa.

Morgan ST. 1995. Life and dead in the plankton: larval mortality and adaptation. En: McEdward L (ed). Ecology of marine invertebrate larvae, pp. 279-322. CRC Press, Boca Raton.

Mutschke E \& C Ríos. 2006. Distribución espacial y abundancia relativa de Equinodermos del Estrecho de Magallanes. Ciencia y Tecnología del Mar 29: 91-102.

Nishizaki MT \& JF Ackerman. 2004. Juvenile-adult associations in sea urchins Strongylocentrotus franciscanus and S. droebachiensis: Is nutrition involved? Marine Ecology Progress Series 268: 93-103.

Ojeda FP \& B Santelices. 1984. Invertebrate communities in holdfasts of the kelp Macrocystis pyrifera from southern Chile. Marine Ecology Progress Series 16: 65-73.

Pennington JT. 1985. The ecology of fertilization of echinoid eggs: The consequences of sperm dilution, adult aggregation, and synchronous spawning. The Biological Bulletin 169: 417-430.

Pennington JT \& RB Emlet. 1986. Ontogenetic and diel vertical migration of a planktonic echinoid larva, Dendraster excentricus (Eschscholtz) occurrence, causes, and probable consequences. Journal of Experimental Marine Biology and Ecology 104: 69-95.

Pennington JT \& RR Strathmann. 1990. Consequences of the calcite skeletons of planktonic echinoderm larvae for orientation, swimming, and shape. The Biological Bulletin 179: 121-133.
Pickard GL. 1971. Some physical oceanographic features of inlets of Chile. Journal of the Fisheries Research Board of Canada 28: 1077-1106.

Pineda J. 1999. Circulation and larval distribution in internal tidal bore warn fronts. Limnology and Oceanography 44: 1400-1414.

Pineda J. 2000. Linking larval settlement to larval transport: Assumptions, potentials and pitfalls. Oceanography of Eastern Pacific 1: 84-105.

Poulin E, AT Palma, G Leiva, E Hernández, P Martínez, SA Navarrete \& JA Castilla. 2002. Temporal and spatial variation in the distribution of epineustonic competent larvae of Concholepas concholepas along the central coastal of Chile. Marine Ecology Progress Series 229: 95-104.

Primus AE. 2005. Regional specification in the early embryo of the brittle star Ophiopholis aculeata. Developmental Biology 283: 294-309.

Scheltema RS. 1986. On dispersal and planktonic larvae of benthic invertebrates: an eclectic overview and summary of problems. Bulletin of Marine Science 39: 290-322.

Shanks AL. 1995. Mechanisms of cross-shelf dispersal of larval invertebrates and fish. En: McEdward L (ed). Ecology of marine invertebrate larvae, pp. 323-368. CRC Press, Boca Raton.

Silva N, HA Sievers \& R Prado. 1995. Características oceanográficas y una proposición de circulación para algunos canales Australes de Chile entre $41^{\circ} 20^{\prime}$ 'S y $46^{\circ} 40^{\prime} \mathrm{S}$. Revista de Biología Marina 30: 207-254.

Sköld M, SR Wing \& PV Mladenof. 2003. Genetic subdivision of a sea star with high dispersal capability in relation to physical barriers in a fjordic seascape. Marine Ecology Progress Series 250: 163-174.

Stotz W. 2003. Sea-urchin fisheries: A Chilean perspective. In: Lawrence JM (ed). Sea urchins fisheries and ecology, pp. 3-17. Destech Publications, Puerto Varas.

Stotz W, S González \& C López. 1992. Siembra experimental del erizo rojo Loxechinus albus (Molina) en la costa expuesta del centro-norte de Chile: efectos del erizo negro Tetrapygus niger (Molina) sobre la permanencia y crecimiento de los juveniles. Investigación Pesquera, (Chile) 37: 107-117.

Thorson G. 1950. Reproductive and larval ecology of marine bottom invertebrates. Biological Reviews of the Cambridge Philosophical Society 25: 1-45.

Valle-Levinson A \& J Blanco. 2004. Observations of wind Influence on exchange flows in a strait of the Chilean inland sea. Journal of Marine Research 62: 721-741.

Valle-Levinson A, F Jara, C Molinet \& D Soto. 2001. Observations of intratidal variability of flows over a sill/ contraction combination in a Chilean fjord. Journal of Geophysical Research 106: 7051-7064. 
Vásquez J. 2001. Ecology of Loxechinus albus. En: Lawrence JM (ed). Edible sea urchins: Biology and ecology, pp. 161175. Elsevier Science, Tampa.

Vásquez J, JC Castilla \& B Santelices. 1984. Distributional patterns and diets of four species of sea urchins in giant kelp forest (Macrocystis pyrifera) of Puerto Toro, Navarino Island, Chile. Marine Ecology Progress Series 19: 55-63.

Wing SR, JL Largier, LW Botsford \& JF Quinn. 1995. Settlement and transport of benthic invertebrates in an intermittent upwelling region. Limnology and Oceanography 40: 316-329.

Wing SR, MT Gibbs \& MD Lamare. 2003. Reproductive sources and sinks within a sea urchin Evechinus chloroticus, population of a New Zealand fjord. Marine Ecology Progress Series 248: 109-123.
Young CM. 1995. Behavior and locomotion during the dispersal phase of larval life. En: McEdward L (ed). Ecology of marine invertebrate larvae, pp. 249-278. CRC Press, Boca Raton.

Zamora S \& W Stotz. 1992. Ciclo reproductivo de Loxechinus albus (Molina 1782) (Echinodermata: Echinoidea) en Punta Lagunillas, IV región, Coquimbo, Chile. Revista Chilena de Historia Natural 65: 121-133.

Zar JH. 1999. Biostatistical analysis, 663 pp. Prentice Hall, Englewood Cliffs.

Zuleta A, C Molinet, PS Rubilar, S Rosales, R Gili, L Ariz, N Barahona, Z Young, B Ernst, JM Orensanz, A Parma \& M Nilsson. 2008. Bases biológicas para la rotación de áreas del recurso erizo, Fase II. Informe Final. Proyecto FIP 2003-13: 1-40. 